\title{
EVOLUTION OF PRIMORDIAL PROTOSTELLAR CLOUDS
}

\author{
K.OMUKAI, R.NISHI, H.UEHARA \\ Department of Physics, Kyoto University \\ Kyoto,606-01,Japan \\ AND \\ H.SUSA \\ Center for Computational Physics, University of Tsukuba \\ Tsukuba,305,Japan
}

The formation of stars in proto-galactic clouds can be viewed as two step processes i.e. the fragmentation of proto-galactic clouds and evolution of these fragments into stars. We consider here the latter process, the contraction of protosteller clouds $\left(\sim 1 M_{\odot}\right)$ which consist of primordial gas. We investigate cooling processes by calculating the radiative transfer of $\mathrm{H}_{2}$ rotational/vibrational lines. We consider clouds in hydrostatic equilibrium as initial conditions. Comparing two timescales, the freefall time $t_{f f}=\sqrt{3 \pi / 32 G \rho}$ and the timescale of quasi-static contraction $t_{q s c}=$ $\rho /\left(\frac{\partial \rho}{\partial t}\right)_{\text {quasi-static }}\left(\sim t_{\text {cool }}\right.$, the cooling time) of these clouds, we find that as the clouds contract, the ratio of two timescales $t_{f f} / t_{q s c}$, i.e. the efficiency of cooling, becomes larger even under the existence of cold and opaque envelope. Especially for the fragments of primordial filamentary clouds $\left(t_{f f} \sim t_{q s c}\right.$ initially), they collapse dynamically in the freefall timescale. This efficiency of cooling is utterly peculiar to the line cooling.

\section{References}

Omukai, K., Nishi, R., Susa, H. \& Uehara, 1997. in preparation

Uehara, H., Susa, H., Nishi, R., Yamada, M.

\& Nakamura, T., 1996. Astrophys.J., 473, L95. 